\title{
FROM DESCRIPTIVISM TO CONSTRUCTIVISM - A CHALLENGE TO SYMBOLIC MODELING. I
}

\author{
ARNE KJELLMAN \\ Dept. of Regional Planning, Royal Institute of Technology, Stockholm \\ Dept. of Information Technology, Mid-Sweden University, Sundsvall. \\ E-mail: Arne.Kjellman@ite.mh.se
}

\begin{abstract}
Classical modeling is not isomorphic, on the contrary the "objects of reality" or the like is the source of a homomorphic mapping performed to produce the model - just the way a piece of landscape is portrayed by its map with some bewildering details left out. We are thus taught that this process of modeling (or abstraction) is a plain mapping procedure - we call this descriptivism or representationalism. The prevailing object-oriented modeling approach - or realistic approach - has some serious shortcomings due to the negligence of some aspects of the observer function, which for instance has resulted in a "world definition" made from "outside" the living consciousness (realism or materialism). By reversing this picture instead taking off from the impressions arisen within the subject's (the observer/knower's) conscious experience - the subject-oriented approach - and ask how a living consciousness organize s itself to handle the task of living, we gain new insights in the process of conceptualization and learning. We learn that the dualistic worldview is superfluous and should better be replaced by a neutral monistic approach, where the hypothetical existence of an independent outside reality (realism) can be substituted by the idea of a reality constructed from inside a living consciousness nothing else but a model whose main purpose is to guide human anticipation and facilitate communication. Taking that stances the main tasks of human consciousness just become modeling - creating the outside model reality and th e inside domain of feelings. In such a framework also the classical truth - in the sense of a God-given modeling truth become meaningless - and must be substituted by the Pierce-ain pragmatical or consensual truth. In the subject-oriented approach states, properties etc. are not given any observer independent existence. On the contrary they emerge at the moment of their measurement as advocated by the Copenhagen interpretation. Bell's theorem also states: Given the quantum mechanics, either the idea of Einstein locality or the idea of an observer independent reality must be abandoned. The subject-oriented approach clearly abandons the idea of a pre-given observer independent reality - in favor of a cognitive agent created private reality, which then become the base for defining an "objective reality" in the form of a consensual scientific agreement.
\end{abstract}




\section{INTRODUCTION}

The main aim of this paper is to introduce the reader to the ideas of the subject-oriented approach of modeling [1,2]. The prevailing object-oriented approach - or realistic approach - has some serious shortcomings due to the negligence of some aspects of the observer function, which for instance has resulted in a "world definition" made from "outside" the living consciousness (realism or materialism). By reversing this picture instead taking off from the impressions arisen within the subject's (the observer/knower's) conscious experience and ask how a living consciousness organizes it to handle the task of living, we gain new insights in the process of conceptualization and learning. We learn that the dualistic worldview is superfluous and should better be replaced by a neutral monistic approach. Many phenomena which are puzzling using the object-oriented approach turns out to be very natural and obvious in the subject-oriented approach - but unfortunately one have to re-orient one's way of thinking considerably to appreciate the new point of view. I regard this reorientation as a possible shift of paradigm - abandoning descriptivism in favor of constructivism. I want the ideas and principles presented in this paper to be accessible to a wide audience and this dictates the rather informal style and the necessary short cuts taken to carry the discussion further on the intuitive level. This must not be mistaken for shallowness or a lack of argument - this is just an effort to present these ideas in a paper decent in size.

\section{REALISM AND SCIENTIFIC OBJECTIVITY}

Let us take off with the base assumption of realism - that there is a reality "out there" which has states, properties and behavior independent of any observer. In this view the real world - or outside reality - is the source of experience and data when doing scientific observations. The observations and our experience are reported in the natural language and in form of tables, pictures etc. We denote such description facts. A fact is most easily is described as a piece of exchangeable human experience. When I say:" Things are like this or that", I say so fully convinced about that the receiver of my message could assure himself, at least principally, that my statement is correct. One way for him to do so, is to gain experience the same way I did it. That is, facts are exchangeable experiences that can be made into shareable knowledge. The individual scientist reports his experiences in a paper that contains a precise description of the situation during which his experience is established - and could as well be established by other researcher according to his opinion. If the original researcher's experience can be repeated by others it is proven - not that his experience is true - but that his experience was exchangeable. This piece of experience is thus made into common scientific knowledge and in this way the individual knowledge is made common property - is made an objective fact. Various facts regarding a specific phenomenon can then be gathered and compiled into models or a set of rules and then visualized in different ways. 
In this way the experience of an individual researcher turns into a model. As a first step, this model is strictly private, but if this model is accepted by other scientists later on (as a scientific agreement) it can turn into a model common to all researchers of that specific scientific area. As advocated by Kuhn this view suggests a consensual scientific practice depending on rather different premises, rather than a dogmatic postulation of an observer-independent reality.

We know that the model has an important role in this process of information interchange. Thus it is of utmost importance that the modeling process is clearly understood by scientists of all disciplines and that the rules and assumptions involved in this process are easily grasped and well known. Unfortunately this is not generally the case. Modeling - or systems modeling - is an activity that requires a conceptual framework within which one operates and for the sake of communication this framework must be similar or inspired by the frameworks currently in use. Models that represent time-dependent systems behavior has in general systems theory and control theory gained a mathematically consistent foundation. The literature on this subject is huge - just turning to systems modeling there are many proposals for formal frameworks $[3,4,5,6,7]$.

\section{THE PREVAILING REALISTIC APPROACH - DESCRIP- TIVISM}

Modeling of systems has its place as a principal tool in all sciences. Models are used for the purpose of description, prediction and control. These are the main domains of discourse within areas as: systems analysis, systems design, control theory, operations analysis, simulation, management information systems, information systems, decision support systems etc. Since modeling in its widest sense is the only means of human communication this narrow technological view must be widened by the recognition that modeling is essential for any human activity - not only scientific ones - and that modeling is not restricted to mathematical modeling only. The importance of models and model building as an integral part of scientific inquiry has often been stated [8]:

- No substantial part of the universe is so simple that it can be grasped and controlled without abstraction. Abstraction consists in replacing a part of the universe under consideration by a model of similar but simpler structure. Models... are thus a central necessity of scientific procedure.

The notation "a part of universe" or equivalent "a part of the real world" is usually denoted as an object or a system. By explicitly representing the knowledge about the components (parts) of a system we specify this knowledge. This specification can be made in many different ways, and they can all be thought of as models. That is a model is to be seen as a system specification developed and expressed in a specific conceptual framework.

There are different definitions of the model conception. Most of them adhere 

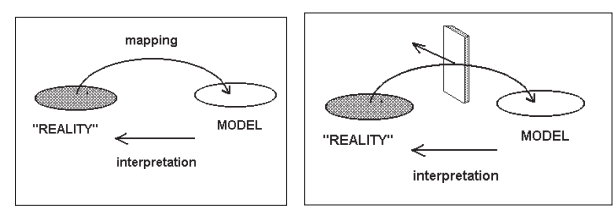

Figure 1.

Figure 2.

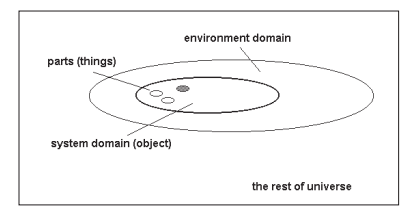

Figure 3.

to a description in which the reality is seen as the source of a mapping into an abstract model. The modeling relation is very often depicted as in fig.1. In this view the objects of reality are mapped into models, taking the "outside" reality for granted. Also the "rules of modeling", i.e., the rules of formalization is often also regarded as self-evident. The inverse mapping occurs when we interpret the "model behavior" in terms of a corresponding "reality" behavior. This mapping procedure is not isomorphic. The "objects of reality" or the like is the source of a homomorphic mapping $[9,10]$ performed to produce the model - just the way a piece of landscape is portrayed by its map - with some bewildering details left out. This homomorphic mapping procedure (abstraction) can be illustrated by inserting a filter in the mapping path fig. 2 - thereby suggesting that the model is a "fade copy" of a richer original. But still a "fade copy" in many respects can be more useful than $t$ he original since it just catches the relevant aspects of the phenomenon. This attitude of modeling we call descriptivism or representationalism.

We can use this view to establish one fact: In the modeling relation the original - the phenomenon under consideration itself "B "- is certainly no model. That is to say, a model "A" is somewhat sort of a "copy" that is separated (or distinct) from the original " $\mathrm{B}$ " in some respect - and this fundamental feature is also the essence of abstraction.

\section{SYSTEMS MODELING AND REAL WORLD VIEWS}

In this classical dualistic view the phenomena of the world are classified as concrete or abstract and the word object is reserved to denote a concrete physical phenomenon (a concrete thing or any limited amount of matter). The word concept is used to denote an abstract phenomenon and sometimes the phenomenon of interest is not a clearly defined object in its physical sense of the word. In this case we use the notion area to designate a part of the world in order to delimit " the scope of interest" or the "domain of analysis". This " area" can be a natural phenomenon (a living cell) or a man-made (a factory) or abstraction (a poem), but we must remember that this distinction has risen in the mind of an observer only. This "area" definition must in such case meet another demand: The description or specification must be done so that it can be uniquely decided if any phenomenon belongs to a specific area or not.

The area of interest - being a physical object or not - is defined by the 
observer in terms of a system domain. The environment domain of a system are such phenomena, which are not part of the system domain, but are necessary in order to describe the systemic properties of the system. The rest of universe is considered of no interest for this particular modeling effort. I must once more emphasize that all these domain definitions are in the mind of an observer - at best guided by some given consensual scien tific agreement.

These suggestions are in line with the formal definitions of ontology [11], and from this source we can add the following: All objects have properties. The objects are "known" by us only through their properties and properties are often represented in terms of attributes. An object can and have normally more properties but attributes, because we normally do not know some properties. Though a known property must have at least one attribute representing it.

To gather the strands to the state space approach we observe that some properties are time varying and others are more permanent. Time-varying properties are often used to define states. The notion state and property is often interchangeable, although in a natural language they may differ in their interpretation. An attribute can in many respects be thought of as a state variable. A system domain always "contains" an unlimited number of phenomena, each of which can be investigated. Every phenomenon is thought to have a large and unknown number of properties and these can be assigned different attributes. To restrict the domain of analysis - by mapping the reality into an abstract system (abstraction) - is necessary just in order to be able to proceed at all. This mapping is carried through by specifying objects (entities), attributes, attribute values and underlying domains etc.

This world view is certainly compatible with the object oriented world view [12] - as a matter of fact any object can be regarded as a system. This point of view is also common in control theory and the similarities of are hinted on in fig 4 . where the environment domain interacts with the system domain vice versa. The environment acts upon the system by the input variables and the system acts upon the environment by the output variables.

The input variables is anything effecting (passing the border of) the system domain, i.e., control signals, material flows, disturbances etc. The output variables are anything leaving (passing the border of) the system domain, i.e., measurement signals, material flows etc. Note that the observer of the system domain belongs to the environment domain and often the observer disturbs the system domain under consideration. This phenomenon is very well known in the social sciences and has fundamental principal implications as stated in Heisenberg's principle of uncertainty.

The block diagram is a basic conceptual tool used in control theory for illustrating functionally the components of a control system. There are two methods to describe mathematically a control system. A description "from outside", which is done by means of the transfer function:

$$
\mathbf{y}=G(s) \mathbf{u}
$$




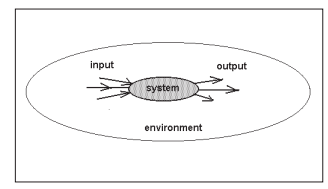

Figure 4.

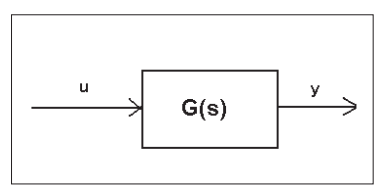

Figure 5.

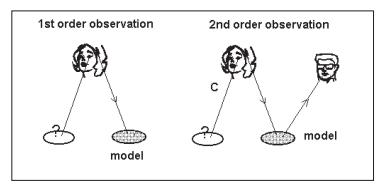

Figure 6.

which states how the output varies as a function of the input. The structure of the system is left without consideration and the transfer function block is then treated as a "black box". The state space approach defines an "inner structure" of the system: the state, which is described by means of a state vector $\mathbf{x}=\left\{x_{1}, x_{2}, x_{3}, \ldots, x_{n}\right\}$. For a linear system is:

$$
\begin{aligned}
d \mathbf{x} / d t & =A \mathbf{x}+B \mathbf{y} \\
\mathbf{y} & =C \mathbf{x}+D \mathbf{u}
\end{aligned}
$$

where $\mathbf{x}, \mathbf{u}, \mathbf{y}$ are state, input and output vectors respectively and in general functions of time and $A, B, C, D$ matrices.

The mentioned formalisms suggest that the state space approach is an appropriate approach then suggesting a complete specification formalism for mathematical modeling of the elements of an independent physical reality. This was the refinement of the Newtonian laws of motion described by differential equations, cast in a more general and elegant form. These became the mathematical foundation (model) of the mechanical paradigm - completely causal and deterministic. The limitations of modeling nature in this w ay soon became apparent - "to set up the equations is one thing, to solve them is quite another" [13]. Exact solutions often were restricted to few simple and regular phenomena. Science developed two methods to mathematically model natural phenomena; the deterministic equations of simple systems (the state space approach) and the statistical equations of thermodynamics (for complex systems). They both featured linear equations. Sometimes non-linear equations emerged but they were too di fficult to solve unless linearized.

The decisive change during the last three decades has been to recognize that nature is relentlessly non-linear. The exploration of non-linear systems has had a profound impact on science as a whole, and it has forced us to reevaluate the relationships about the mathematical model and the phenomena it describes. This progress was mainly due to the modern computer and its capacity to solve non-linear equations and simulate non-linear phenomena. The feedback loop and 2nd order cybernetics [14] also revealed the importance of self-organization, which was developed in the theory of "dissipative structures" [15]. This theory deals with open systems far from equilibrium, characterized by non-linear equations, where even simple systems can display a chaotic behavior. 
The new mathematics of complexity and the concept of self-organization launched the idea of the pattern of organization as the clue to the understanding of complexity - but the one-sided object-oriented approach was never questioned in spite of the emergence of quantum mechanics in the early 1920ties.

From the other side cybernetics and social systems theory started to pay interest in self-organization and self-referential systems pointing out the essence of the observer's role when defining a system. These early indications has been emphasized by the cognitive sciences interest in perception, cognition and consciousness and this interest has also revealed a row of phenomena which cannot be explained within the materialistic framework in its classical sense.

\section{CRITIQUE OF THE CLASSICAL VIEW}

The presented and prevailing object-oriented approach (the realistic approach) - has some serious shortcomings due to the negligence of some aspects of the observer function. Another even riskier undertaking is the questionable definition of an observer-independent reality, which has resulted in a "world definition", made from "outside" the living consciousness (realism or materialism).

Let us first observe that traditional modeling is performed from a 2 nd order observer's viewpoint, unfortunately disregarding the intermediate link of the observer $C$ (the subject) itself.

It is fairly easy to understand that what is primarily modeled in this situation is not any "outside" reality but instead the percept rising in the mind of an observer (the $I$-reality). It is also important to realize that this process is not a direct one-step mapping of an "outside" reality into a model but rather must be seen as a two-step procedure:

1) - a "projection" of an object of reality into the mind of the scientist - as a percept (a perceptual path)

2 ) - the lays out (construction) of this percept into a system model (a conceptual path).

To circumvent the shortcomings of the more traditional model definitions, we can use the functional model definition proposed by Marwin Minsky [16]:

An object " $A$ " is a model of an object " $B$ " for an observer " $C$ ", if observer can use " $A$ " to answer questions that interest him about " $B "$.

This view regards any model as a base of data (DB) or knowledge (KB) and also takes the observer of the system in consideration. This is essential. Furthermore this definition mentions the purpose of the observer and such a purpose has certainly as a heavy influence on the outcome of the modeling process. This definition underlines the fact that every model is associated with and restricted by the way it is represented (laid out). The percept formed in the mind of the system viewer (the I-reality) is obviou sly much more rich but the resulting model and is usually termed a mental re-presentation of the 
objects of reality or the system under consideration. Such a view is misleading and I will later argue that this is no re-presentation at all.

Any system is an abstraction - in spite of the fact that the normal use of the term system - "a concept that captures a part of the real world" suggests that this act of capturing could be accomplished objectively in some sense. This is a misconception. Any system definition is an abstraction - in the first stage very private. In contrast the definition of a physical object is never thought of as an abstraction. This is also a misconception I will claim - the subjectoriented approach claim that every system and object definition is at first subjective and is always defined relative to some observer - a point of view that hitherto has been muddled by the rigid - and unnecessary - materialistic world definition. To circumvent the traditional view regarding the reality as given beforehand (real) and observer-independent it is wise to take one step beyond the modeling of percepts - to involve in the modeling of ideas.

\section{THE MODELING OF IDEAS}

Let us contemplate the conception of a "point". We ask what comes to the mind when we encounter the "point" conception? The idea of a point - is a probable "explanation". This idea is abstract - since the generative process behind the rise of the "point" idea does not involve the senses. In spite of this fact we can probably not build up the relevant knowledge to properly apprehend the conception of a point without the use of our senses (our experience) - the immediate rise of the idea does not involve the use of senses. I am convinced that every normal human being can form the idea of a "point" and contemplate this abstract idea just like the way that I do.

I believe in this - until the point of certainty - but how should I know for certain? In a communicative context I could ask the person in question: "Describe a point!" or ask him to indicate a "point" - but until the day we have any means to more directly communicate the content of human consciousness - we certainly have no other option but to ask the person.

We ask $C$ : "What is a point?" $C$ could answer: "A point is a dot that does not occupy any space" - an utterance. The source of this utterance is $C^{\prime} s$ idea - for the moment disconnected to any perceptual input - and to establish consensus we as receivers must be able to interpret and agree on that utterance. The utterance of $C$ is an action namely the "effort to utter" - and the result thereof is a sentence - or a verbal model. Since an abstract phenomenon is space-less (non-perceptible) we might need a clearer explanation. $C$ says: "A very small dot" - but also such explanation needs an action and this could result in a drawn dot - i.e., the model point. $C$ could also hit the point-marked key on the computer keyboard - clearly an action. $C$ could also point to a grain of sand and say: "This is a close to a point" another iconic model.

There are many ways to communicate the idea of a "point" but they all have in common that this cannot be done without an action - and without the 
result thereof. That idea can be made into a principle, we cannot break the introvert world of ideas without outward actions. The resulting manifestation of this act is a "physical construct" - a model. This model is as a first stage nothing else but a private model (a personal sign or reminder) of the original idea or mental construct.

Mostly the phenomena of reality are explicitly presented modeled by one model only. This is a simplification. In practice we observe the obvious need of many different models to frame a certain idea presented - such an attitude is called multi-facetted modeling [17]. We then as a rule use a row of different models in parallel for the purpose of presenting an idea - even if this fact is seldom recognized in science. For instance iconic, verbal, mathematical, logical, conceptual models etc. can be (but seldom ar e) laid out together each catching some aspects of the behind lying idea. In fact any human model is accompanied by a verbal model - a spoken or written description thereof so human modeling efforts are always and has always been multi-facetted in that respect.

\section{THE CATEGORIZATION OF MODELS}

When it comes to the categorizing models we can make use of Peirce's sign trichomomy - symbols (linked to the original by cultural conventions), icons (linked by similarity) and indices (linked physically). In such a view we call models based mainly on the concatenation of common cultural symbols symbolic models. A symbol is an element $a_{i}$ drawn from an alphabet $T=$ $\left\{a_{1}, a_{2}, a_{3}, \ldots, a_{n}\right\}$ - a set of marks chosen according to some cultural or scientific convention. The symbols of our language as the most obvio us example and $T=\{0,1\}$ - the binary alphabet - as the minimal useful set. Among the symbolic models we find the verbal model (the written or spoken natural language), the mathematical model and other formalized languages, logic, predicate logic, the conceptual model and many others.

The iconic models are based on a visual similarity (models focusing on a functional similarity are called analogical). The pencil and paper, which was the useful output device of Newton's mathematics, is nowadays superseded by the computer screen, which has extraordinary graphical possibilities as exposed. Among the iconic models we find small-scale models of different kinds, the map, the photo, the mannequin, works of art etc.

\section{THE MODELING FRAMEWORK - A LANGUAGE}

When the mentioned introvert-breaking act of physical construction has become a habit - the modeler/constructor has established a modeling framework (or a language) still of strictly personal use. In this situation the basic concepts of the model are familiar to the constructor (i.e., induces interpretable percepts in its conscious experience) and therefore they are the signs upon which the constructor can erect useful interpretations. 
The purpose of this act of description is not only interpersonal communication, since we recognize the need of auto-communication - a physical act outwards to "shape our own intellectual understanding" of a concept or an idea. We often make use of such auto-communicational acts involving physical sign production in order to "sharpen our understanding" of a concept for instance when sketching on ideas in a notebook or the like. A process of chiseling out useful concepts from fuzzy ideas.

How do we proceed in order to communicate our ideas to our fellow beings? Since telepathy is not possible, there is no other option but to teach fellow beings to become outside interpreters by learning rules of the modeling framework and how to interpret different concepts. This learning process must establish a connection between the ideas of the model constructor and the personal ideas of the learner. That is to say, assuming that the learner carries the fundamental idea of "a point" in its imagination - the "model ink dot" has to be connected to this very "point idea". Thus the physical appearance of my construction - the model point - must in due time act as a reliable trigger of the "point idea" of the learner. Any sketch, drawing, mathematical model and any other model has precisely that function - to trigger the appropriate interpretation procedures within the receiver. Piaget suggests such development of the child's notion of an "object" [18].

In due time we must include a whole scientific discipline or culture in this act of education. As the end result we will at best have a modeling framework (a language or a set of cultural conventions) which will serve as a tool of intersubjective communication and form the basis of intersubjective understanding, i.e., a language. In this context I use the terms language and models in its widest possible sense - including icons, drawings, aesthetic texts, music, tactile communication etc. All frameworks of $\mathrm{m}$ odeling are communication tools in that respect and they all make use of some medium that is able to present sensory stimuli to the receiver. Multimedia presentations are the result of multifaceted modeling in that sense. Science has been regarded superior when building modeling frameworks - take mathematics for an example - but it certainly comes to the very definition of concepts and rules used to build up the modeling framework in question to claim any form of superiority. Science has clearly has no right to claim superiority just in terms of some diffuse "objectivity" claim.

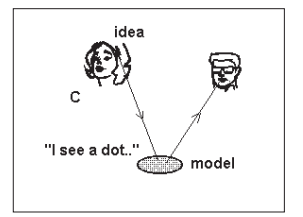

Figure 7.

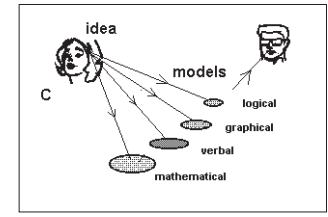

Figure 8.

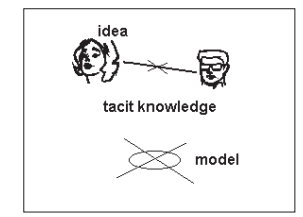

Figure 9.

Some experience - tacit knowledge [19] - is impossible to express in the form of a model - since this experience by definition impossible to concep- 
tualize. The taste of a wine for instance come close to such attribution not to speak about unconscious human experience - which is generally mediated by hints and unconscious gestures. By acting and living together people can exchange tacit knowledge and thus get involved in non-formalized communication. These are "languages" with very vague symbols and lacking a k nown syntax - still containing semantics to the initiated and such knowledge cannot clearly be acquired by the book. We often refer to such knowledge by the term's intuition and the feeling of fingertips. There is large amount of experiential phenomena which we today are unable to frame within a working scientific modeling framework and this also emphasize the need of both a multi-model framework and the use of even "nonscientific" approaches when we try to frame scientific phenomena.

To stress what is said we notice that the scientific "idea of a point" is not entirely caught in the above-mentioned constructive acts, as we also consider a point to be a mathematical abstraction - closer stated a geometrical one. Mathematics is considered a game that can be played without any regard to "reality" - but nevertheless the idea of a point evidently bears on "the experience of living". In this context a "point" has another interpretation that must be caught. A point has no extension, no structure and is consequently indivisible - and its function is reference. A point of reference in space-time - an extension of man's index finger - the definition of location in a spacetime-frame - or an anchor point of the presumed "things of reality".

This brings us to the other central question of this paper - if there really are any such predefined objective "things"- and if so - could we intellectually grasp any essence contained in these? In the case of a mathematical "point" of course there is not much to grasp - since such a point is almost a total abstraction - an "physical" object peeled off all its inherent qualities or properties.

\section{REFERENCES}

[1] von Glaserfeld, E., Radical Constructivism, The Falmer Press, London, 1995.

[2] Maturana, H.R., "Ontology of Observing", Am. Society For Cybernetics Conf., Felton, CA, 1988

[3] Klir, G.J., An Approach to General Systems Theory, Van Nostrand Reinold, NY. 1969.

[4] Padulo, L., and Arbib, M.A., Systems Theory: A Unified State Space Approach to Continuous and Discrete Systems. W.B.Saunders, Philadelphia,PA,1974.

[5] Mesarovic, M.D., Takahara, Y. General Systems Theory: Mathematical foundations.Academic Press, New York, NY, 1975.

[6] Zeigler, B., Multifacetted Modeling and Discrete Event Simulation, Academic Press, 1984.

[7] Wymore, A.W., A Mathematical Theory of Systems Engineering: The Elements. Krieger Publishing Co., 1977

[8] Rosenbluth, A. and Wiener, N., The Role of Models in Science, Philosophy of Science XII, No 4, Okt. 1945

[9] Rosen, R., Life Itself, Colombia Univ. Press, NY. 1991 
[10] Casti, J. L., Alternate Realities, John Wiley \& Sons, NY.1988.

[11] Bunge, M., Treatise on Basic Philosophy: Vol.3 Ontology I: The Furniture of the World, Reidel, Boston, 1977.

[12] Rumbaugh, J., Blaha, M., Premerlani, W., Frederick, E., and Lorenson, W., ObjectOriented Modeling and Design. Prentice Hall, 1991.

[13] Stewart, I., Does God Play Dice., Penguin Books, 1990.

[14] Foerster, H. and Zopf, G. (eds.) Principles of Self-Organization, Pergamon, New York, 1962.

[15] Priogine, I. , From Being to Becoming, Freeman, San Fransisco, 1980.

[16] Minsky, M. L., "Matter, Mind and Models", Proc. of IP Congress, Vol 1, Spartan Books, pp. 45-49, 1965

[17] Zeigler, B., Multifaceted Modelling and Discrete Event Simulation, Academic Press, 1984.

[18] Piaget, J., La construction du rel chez les enfants, 1938 (The construction of reality in the child, Trans. M.Cook, New York, Basic Books, 1971)

[19] Polanay, M., The Tacit Dimension, Routledge \& Kegan Paul Ltd, xxx, XXX. 